See discussions, stats, and author profiles for this publication at: https://www.researchgate.net/publication/279909529

\title{
Matching-To-Sample and Respondent-Type Training as Methods for Producing Equivalence Relations: Isolating The Critical Variable
}

Article in The Psychological record · July 2001

DOI: 10.1007/BF03395407

\section{CITATIONS}

69

2 authors, including:

\section{Geraldine Leader}

National University of Ireland, Galway

94 PUBLICATIONS 2,454 CITATIONS

SEE PROFILE
READS

56

Some of the authors of this publication are also working on these related projects:

Application of Applied Behavior Analysis to the Management of Gestational Weight Gain and Investigation into Weight

Mediated Conditions as Risk Factors for the Development of Autism Spectrum Disorder in Offspring View project

GEMMA (Genome, Environment, Microbiome and Metabolome in Autism View project 
Matching-to-sample and respondent-type training as methods for ...

Leader, Geraldine;Barnes-Holmes, Dermot

The Psychological Record; Summer 2001; 51, 3; Social Science Premium Collection pg. 429

The Psychological Record, 2001, 51, 429-444

\author{
MATCHING-TO-SAMPLE AND RESPONDENT-TYPE \\ TRAINING AS METHODS FOR PRODUCING EQUIVALENCE \\ RELATIONS: ISOLATING THE CRITICAL VARIABLE
}

\author{
GERALDINE LEADER \\ University of UIster at Jordanstown
}

\author{
DERMOT BARNES-HOLMES \\ National University of Ireland, Maynooth
}

\begin{abstract}
The purpose of this study was to compare systematically the effectiveness of the respondent-type training procedure and the matching-to-sample training procedure. In Experiment 1, a withinsubject design was used, to compare the effectiveness of the two procedures. In Condition 1, students were trained using the respondent-type training procedure (60 training trials) and tested for the emergence of symmetry and equivalence responding using a matching-to-sample test. Students were subsequently trained using the matching-to-sample training procedure (60 training trials) and tested using a matching-to-sample test. In Condition 2, the order of the training and testing was reversed (i.e., i, MTS training; ii, MTS test, iii, respondent training; iv, MTS test). Experiment 2 was identical to Experiment 1, except that during matching-to-sample training subjects were required to produce 12 consecutively correct responses before an equivalence test. During respondent-type training students were presented with 12 training trials. Experiment 3 was identical to Experiment 2 except that the two negative comparisons were removed from matching-to-sample training. Experiment 4 was identical to Experiment 3 except that the correct comparison appeared to the right, center, or left of the screen and three response keys were used. In Experiments 1,2, and 3 respondent-type training was more effective than matching-tosample training. In Experiment 4 when the negative comparisons were removed from matching-to-sample training and when the spatial position of the correct comparison varied both procedures were equally effective.
\end{abstract}

Researchers studying the phenomenon of stimulus equivalence typically use a matching-to-sample (MTS) procedure (Sidman, 1971, $1980,1986,1987)$. In a typical experiment, for example, six MTS tasks may be used during the training phase to establish related conditional

This research was conducted as part of Geraldine Leader's doctoral research program under the supervision of Dermot Barnes-Holmes. These data were presented at the Symposium on Stimulus Equivalence at the Annual European Meeting of the Experimental Analysis of Behavior Group, London, April 1996. Requests for reprints may be obtained from Dermot Barnes-Holmes, Department of Psychology, National University of Ireland, Maynooth, Co. Kildare, Ireland. (Dermot.Bames-Holmes@may.ie). 
discriminations. A subject may be taught to pick Comparisons $B 1$ and $C 1$ given the Sample Stimulus A1, B2 and $C 2$ given $A 2$, and $B 3$ and $C 3$ given A3. On each of these tasks, a single sample stimulus is presented along with three comparison stimuli and the subject is required to choose one of the comparisons. For example, during training, choosing Stimulus B2 when $\mathrm{A} 2$ is presented as a sample produces positive feedback (e.g., "Correct" appears on the screen). However, choosing B1 when A2 is presented as sample produces negative feedback (e.g., "Wrong" appears on the screen). After a subject has reached a predetermined mastery criterion, he or she is exposed to an equivalence test (MTS procedure without feedback). Subjects may then be tested for the emergence of symmetry $(B 1 \rightarrow A 1, B 2 \rightarrow A 2, B 3 \rightarrow A 3)$ and combined symmetry and transitivity $(\mathrm{C} 1 \rightarrow \mathrm{B} 1, \mathrm{C} 2 \rightarrow \mathrm{B} 2, \mathrm{C} 3 \rightarrow \mathrm{B} 3)$. This performance is normally referred to as responding in accordance with equivalence relations (see Barnes, 1994; Fields \& Verhave, 1987; Hayes, S. C., 1991).

In one recent study a respondent-type ${ }^{1}$ training procedure was used to produce equivalence responding instead of the typical matching-to-sample preparation (Leader, Barnes, \& Smeets, 1996). During the respondent training procedure nine nonsense syllables were presented to the subject in the form of six stimulus pairs. The first stimulus of each pair simply appeared on the screen for $1 \mathrm{~s}$ (e.g., A1). The screen subsequently cleared for $0.5 \mathrm{~s}$ (within-pair delay) before the second stimulus of the pair (i.e., B1) appeared for $1 \mathrm{~s}$. A 3-s interval (between-pair delay) then occurred before the next stimulus pair was presented in the same fashion. Subjects were not required to demonstrate any overt response during training, but they were simply instructed to look at the computer screen.

All six stimulus pairs (A1-B1, A2-B2, A3-B3, B1-C1, B2-C2, B3-C3) were presented in this way in a quasi-random order for 60 trials, the only constraint being that each stimulus pair was presented once in each successive block of 6 trials. (i.e., each stimulus pair was presented 10 times). When all stimulus pairs were presented subjects were tested for the emergence of symmetry and equivalence relations using a standard MTS test. The vast majority of subjects (84\%) successfully passed the equivalence test.

One interesting issue that arose from this research was the relative ease with which the respondent training procedure produced equivalence responding. Although matching-to-sample has produced very successful outcomes (e.g., Sidman \& Cresson, 1973; Sidman \& Tailby, 1984), some studies have shown this procedure to be less effective (e.g., Fields,

\footnotetext{
1 Consistent with our previous publications in this area, we have included the suffix "type" to indicate that the respondent training procedure described in this article differs considerably from traditional respondent conditioning experiments. For example, the respondent-type training procedure presents nine conditioned stimuli (i.e., nonsense syllables) in various sequences, whereas a typical respondent conditioning experiment presents one or two conditioned stimuli and an unconditioned stimulus. We stress that using the term respondent-type does not imply that the main behavioral process produced by this procedure is best characterized as respondent behavior. For ease of communication, however, the suffix, "type" will not be used in the remaining text.
} 
Adams, \& Verhave, 1993; Saunders \& Green, 1999). The purpose of the present series of experiments was to analyze systematically the relative effectiveness of the respondent versus matching-to-sample training procedures in producing equivalence responding in human adult subjects. In Experiment 1 of the current study, a within-subject design was used, to compare the effectiveness of the two procedures. In Condition 1 , students were trained using the respondent training procedure $(60$ training trials) and tested for the emergence of symmetry and equivalence using a matching-to-sample test. Students were subsequently trained using the matching-to-sample training procedure (60 training trials) and tested using a matching-to-sample test. In Condition 2, the order of the training and testing was reversed (i.e., i, MTS training; ii, MTS test; iii, respondent training; iv, MTS test).

\title{
Experiment 1
}

\section{General Method}

\section{Subject}

Subjects were 6 students, 4 female and 2 male, of University College Cork. Their ages ranged from 18 to 25 . All subjects were experimentally naïve and were nonpsychology majors. Subjects were randomly allocated to one of two experimental conditions.

\begin{abstract}
Apparatus
Subjects were seated at a table in a small experimental room ( $2 \mathrm{~m}$ by $2 \mathrm{~m}$ ) containing an Apple Macintosh SE Microcomputer that displayed black characters on a white background. The computer presented all characters in 12-point New York font. Stimulus presentation and the recording of responses were controlled by the computer, which was programmed in BBC BASIC. The Z, V, and M keys were marked with white paper dots to designate them as response keys. A pool of nine nonsense syllables (CUG, ZID, VEK, YIM, DAX, PAF, ROG, MAU, JOM) were randomly assigned to their respective roles as sample and comparison stimuli for each subject in the study. In the interest of clarity the alphanumerics A1, A2, A3, B1, B2, B3, C1, C2, C3 are used (subjects were not exposed to these labels).
\end{abstract}

\section{Procedure}

Experiment 1 was divided into two conditions, and each condition was divided into four phases. In Condition 1, subjects were exposed to the respondent training procedure followed by a MTS equivalence test, and then subjects were presented with the MTS training procedure followed by a second MTS test. Condition 2 was the same, except that the MTS and respondent training were presented in reverse order (i.e., i, MTS training; ii, equivalence test; iii, respondent training; iv, equivalence test).

At the beginning of Condition 1, subjects were presented with the following instructions: 
In the first stage of this experiment your task is to simply watch the screen.

During the respondent training nine nonsense syllables were presented to the subjects in the form of six stimulus pairs. The stimulus pairs were; $\mathrm{A} 1 \rightarrow \mathrm{B} 1, \mathrm{~B} 1 \rightarrow \mathrm{C} 1, \mathrm{~A} 2 \rightarrow \mathrm{B} 2, \mathrm{~B} 2 \rightarrow \mathrm{C} 2, \mathrm{A3} \rightarrow \mathrm{B} 3, \mathrm{~B} 3 \rightarrow \mathrm{C} 3$. Each stimulus pair was presented in the following sequence. The first stimulus of each pair was presented for $1 \mathrm{~s}$ (e.g., A1), and the computer screen was then cleared for $0.5 \mathrm{~s}$ (i.e., the within-pair delay). The second stimulus (i.e., B1 always followed $A 1$ ) was then presented for $1 \mathrm{~s}$ and the screen was then cleared for $3 \mathrm{~s}$ (i.e., the between-pair delay). Following the 3-s betweenpair delay the next stimulus pair was presented (e.g., B3 $\rightarrow$ C3). Stimuli were always presented in the same position at the center of the computer screen. All six stimulus pairs were presented in this fashion in a quasirandom order for 60 trials, the only constraint being that each stimulus pair was presented once in each successive block of 6 trials (i.e., each stimulus pair was presented 10 times). When all 60 trials had been presented, the screen went blank for $5 \mathrm{~s}$; the equivalence testing instructions then appeared immediately on the screen.

That is the end of the first stage of the experiment. In the next stage, you must look at the nonsense syllable at the top, and then choose one of the three nonsense syllables at the bottom, by pressing one of the marked keys on the keyboard. To choose the left syllable, press the marked key on the left. To choose the middle syllable, press the marked key in the middle. To choose the right syllable, press the marked key on the right.

Press the space-bar twice to continue.

During this stage of the experiment the six symmetry relations (B1 $A 1, B 2-A 2, B 3-A 3, C 1-B 1, C 2-B 2, C 3-B 3)$ and the three combined symmetry and transitivity relations (C1-A1, C2-A2, C3-A3) were tested using the MTS procedure. On each trial, the sample stimulus was always presented in the center, top half of the computer screen $(5 \mathrm{~cm}$ from the upper edge). The three comparison stimuli appeared $1.5 \mathrm{~s}$ after the sample stimulus; no overt observing response was required and the sample remained on the screen with the comparison. The comparisons were presented in a line, $3 \mathrm{~cm}$ from the lower edge of the screen. One comparison appeared $6 \mathrm{~cm}$ to the left, one $6 \mathrm{~cm}$ to the right, and the third directly below the sample stimulus. The location of the comparison stimuli was counterbalanced across test trials. Subjects were instructed at the beginning of the experiment to select a comparison by pressing one of the three designated keys (see instructions above). When a comparison had been selected, the screen cleared immediately and remained blank for $3 \mathrm{~s}$. The next MTS test trial was then presented (i.e., no feedback was presented during the equivalence test). The nine MTS tasks were presented in a quasi-random order for 90 trials, the only constraint being 
that each of the nine tasks occurred once within each block of 9 trials (i.e., each MTS task was presented 10 times; 60 symmetry test trials and 30 equivalence test trials). The stability criterion required subjects to choose the same but not necessarily correct comparison at least 9 times out of 10 on each of the 9 tasks. If subjects produced an inconsistent performance (i.e., less than 9/10 "same responses" on any of the tasks), they were immediately reexposed to the entire experimental procedure. Subjects were administered a maximum of six exposures, at which they either achieved the stability criteria or completed their participation in the study, and they were fully debriefed. Although the stability criteria allowed subjects to proceed if they produced a consistent but incorrect response, the stability criteria for correct responding required the subjects to produce a correct response 9 times out of 10 on each of the 9 tasks.

That is the end of the second stage of the experiment. In the next stage, you must look at the nonsense syllable at the top, and then choose one of the three nonsense syllables at the bottom, by pressing one of the marked keys on the keyboard. To choose the left syllable, press the marked key on the left. To choose the middle syllable, press the marked key in the middle. To choose the right syllable, press the marked key on the right.

Press the space-bar twice to continue.

Students were then presented with a MTS training procedure, during which the following conditional discriminations were reinforced $A 1-B 2$, $\mathrm{A} 2-\mathrm{B} 3, \mathrm{~A} 3-\mathrm{B} 1, \mathrm{~B} 1-\mathrm{C} 2, \mathrm{~B} 2-\mathrm{C} 3, \mathrm{~B} 3-\mathrm{C} 1$. The sample and comparison stimuli remained on the screen together and no observing response to the sample was required. Subjects were instructed at the beginning of this phase to select a comparison by pressing one of the three designated keys (see instructions above). After a correct response the screen cleared and "Correct" appeared on the screen for $1.5 \mathrm{~s}$, accompanied by a high pitched tone. If a response was incorrect the screen cleared and "Wrong" appeared on the screen for $1.5 \mathrm{~s}$. Each training trial was followed by an intertrial interval of $3 \mathrm{~s}$ (the screen remained blank).

All six stimulus combinations were presented in this fashion in a quasi-random order for 60 trials, the only constraint being that each combination was presented once in each successive block of 6 trials (i.e., each combination was presented 10 times). The location of the comparison stimuli was counterbalanced across training trials. Unlike the standard MTS procedure, subjects were not trained to reach a specific success criterion; instead they were simply presented with 60 training trials, and no more. This noncriterion training procedure was deliberately used so that we could compare the MTS training with the respondent-type training reported in the Leader et al. (1996) study. Subjects were simply exposed to 60 training trials in which each stimulus pair (e.g., A1-B1, A2B2) was presented 10 times in a block of 60 trials (i.e., no performance criterion was employed). It was important, therefore, that the matching-to- 
sample training used in the current study replicated this procedure and simply presented 60 training trials. After the final conditional discrimination training trial, the subject was presented with the same equivalence test employed in Phase 2, where the following relations were tested; B1-A3, B2-A1, B3-A2, C1-B3, C2-B1, C3-B2, C1-A2, C2-A3, C3$A 1$. In effect, the stimulus pairs presented during the respondent training did not correspond with the reinforced stimulus pairs presented during the MTS training.

\section{Results and Discussion}

Percent correct on all test trials and mean percent correct calculated across all exposures is presented in Tables 1 and 2 . Of 6 subjects, 3

Table 1

Respondent Training and Testing Followed by Matching-To-Sample Training and Testing

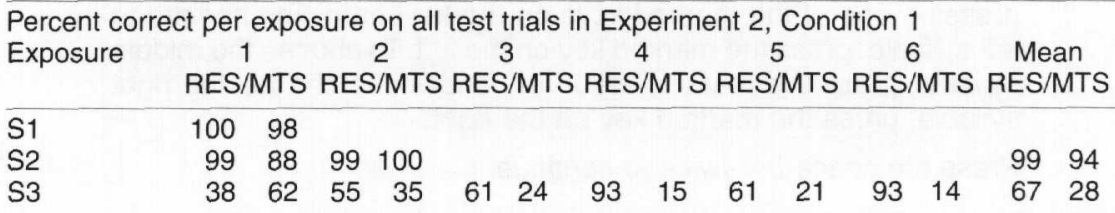

Table 2

Matching-To-Sample Training and Testing Followed by Respondent Training and Testing

\begin{tabular}{|c|c|c|c|c|c|c|c|c|c|c|c|c|}
\hline \multicolumn{13}{|c|}{ Percent correct per exposure on all test trials in Experiment 2, Condition 2} \\
\hline \multicolumn{3}{|c|}{$\begin{array}{l}1 \\
\text { Exposure } \\
\text { MTS/RES }\end{array}$} & \multicolumn{2}{|c|}{$\stackrel{2}{2}$} & \multicolumn{2}{|c|}{$\stackrel{3}{M T S / R E S}$} & \multicolumn{2}{|c|}{$\stackrel{4}{4} \stackrel{5}{M T S / R E S}$ MTS/RES } & \multicolumn{2}{|c|}{$\begin{array}{c}6 \\
\text { MTS/RES }\end{array}$} & \multicolumn{2}{|c|}{$\begin{array}{l}\text { Mean } \\
\text { MTS/RES }\end{array}$} \\
\hline & & & 30 & & & & & 5410 & & & & \\
\hline & 2 & 4 & 40 & 7 & 6 & 10 & 68100 & 60100 & 60 & 100 & & 00 \\
\hline 6 & 57 & 50 & 53 & 73 & 98 & 99 & 98100 & & & & 76 & 80 \\
\hline
\end{tabular}

produced a greater number of correct responses in favor of respondent training (Subjects 3,4 , and 5). The 3 remaining subjects $(1,2$, and 6 ), while failing to produce a response pattern in favor of respondent training, also failed to produce a clear result in favor of MTS training. On their final exposures to the equivalence tests, all 3 responded in accordance with both the respondent and MTS training (i.e., they changed their test patterns dependent upon the type of training that immediately preceded the current equivalence test). In summary, the data from Experiment 1 tentatively suggest that the respondent training procedure more readily facilitated equivalence responding than did the MTS training. 


\section{Experiment 2}

One possible criticism of Experiment 2 might be that the MTS training used here departed somewhat from the standard procedures reported in the equivalence literature. Specifically, in both experiments subjects were exposed to a "criterion-independent" form of MTS training, that required that they complete exactly 60 training trials, irrespective of performance accuracy, before moving onto the next stage of the experiment. In a typical equivalence study MTS training continues until the subject produces a predetermined number of consecutively correct responses. For example a subject may be required to produce 6 out of 6 correct responses in a row, before progressing to the testing phase (e.g., Dymond \& Barnes, 1994). Other studies have required subjects to produce 8 out of 8 correct responses in a row (Healy, Barnes, \& Smeets, 1998; Roche, Barnes, \& Smeets, 1997) or 15 correct responses in a row (Wulfert, Dougher, \& Greenway, 1991). In this experiment subjects were required to produce 12 out of 12 correct responses in a row before progressing to the testing phase. Experiment 2 replicated Experiment 1, except that (a) for the MTS training each subject was required to produce 12 consecutively correct responses before an equivalence test, and (b) only 12 trials were presented during each exposure to the respondent training. The number of consecutively correct MTS training trials was thus matched to the number of respondent training trials.

\section{Subject}

Subjects were 6 students, 3 female and 3 male, of University College Cork. Their ages ranged from 18 to 25 . All subjects were experimentally naïve and were nonpsychology majors. Subjects were randomly allocated to one of two experimental conditions.

\section{Procedure}

Experiment 2 was divided into two conditions. In Condition 1 each subject was presented with the respondent procedure, where each subject received 12 stimulus-pair presentations. The subject was then presented with a MTS test. Subsequently the subject was presented with the MTS procedure, where each subject was required to produce 12 consecutively correct responses prior to an equivalence test. When this had been achieved the subject was presented with the MTS equivalence test. Condition 2 was identical to Condition 1, except the procedure was reversed (i.e., i, MTS training; ii, equivalence test; iii, respondent training; iv, equivalence test).

\section{Results and Discussion}

Percent correct on all test trials and mean percent correct calculated across all exposures is presented in Tables 3 and 4 . All 6 subjects produced a greater number of correct responses in favor of respondent 
Table 3

Respondent Training and Testing (12 stimulus pair presentations) Followed by Matching-To-Sample Training and Testing (12 in a row correct)

\begin{tabular}{|c|c|c|c|c|c|c|c|c|c|c|c|c|c|c|}
\hline \\
\hline \multicolumn{15}{|c|}{ 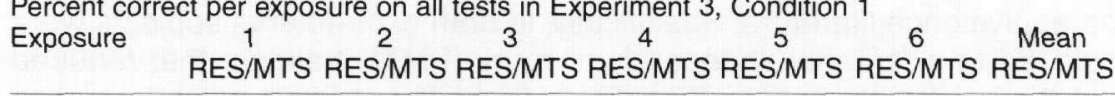 } \\
\hline- & 45 & 12 & 44 & 0 & 48 & 0 & 100 & 0 & 100 & 0 & 100 & 10 & & 4 \\
\hline S8 & 37 & 34 & 47 & 8 & 99 & 33 & 100 & 24 & 100 & 0 & 100 & 0 & 80 & 16 \\
\hline S9 & 42 & 33 & 43 & 21 & 34 & 5 & 63 & 8 & 100 & 10 & 100 & 12 & 64 & 15 \\
\hline
\end{tabular}

Table 4

Matching-To-Sample Training and Testing (12 in a row correct) Followed by Respondent Training and Testing (12 stimulus pair presentations)

Percent correct per exposure on all tests in Experiment 3, Condition 2

\begin{tabular}{lccrrrrrrrrrrrr} 
Exposure & \multicolumn{1}{c}{$\begin{array}{c}1 \\
\end{array}$} & MTS/RES & MTS/RES & MTS/RES & MTS/RES & MTS/RES & MTS/RES & MTS/RES \\
\hline S10 & 3 & 86 & 3 & 90 & 3 & 98 & 0 & 100 & 33 & 100 & 30 & 100 & 12 & 96 \\
S11 & 8 & 80 & 10 & 95 & 12 & 100 & 30 & 100 & 40 & 100 & 25 & 100 & 29 & 91 \\
S12 & 3 & 60 & 10 & 70 & 8 & 75 & 12 & 80 & 20 & 100 & 35 & 100 & 15 & 81
\end{tabular}

training. In Condition 1 and 2 all subjects produced 100\% correct responding during the respondent equivalence test. During the final MTS equivalence test, Subjects 7,8 , and 9 produced $10 \%, 0 \%$, and $12 \%$ correct responding, respectively, while Subjects 10,11 , and 12 produced $30 \%, 25 \%$, and $35 \%$ correct responses, respectively.

In Experiment 2 the respondent procedure was clearly more effective than the traditional MTS procedure. When subjects were required to produce a predetermined mastery criterion of 12 out of 12 correct responses during MTS training, and when subjects were presented with only 12 training trials during respondent training, they performed in favor of respondent training. Indeed, upon closer inspection of the raw data it appears that subjects "adopted" respondent training during the MTS test. In effect subjects responded in accordance with the respondent training during the MTS testing (see General Discussion, p. 20).

\section{Experiment 3}

The results of Experiment 2 raise an interesting question. What makes the respondent procedure more effective, at least in the context of the current study, than the traditional MTS method? One explanation might be that the MTS procedure is "overly complicated" compared to the more simplified temporal pairing procedure of the respondent training method. Experiment 3 was designed to test this suggestion.

In this experiment, during MTS training the negative comparisons were removed from training. That is, the sample appeared on the screen followed by the correct comparison. The subject was then required to make a 
response, (i.e., press the key "V"). The word "Correct" appeared on the screen with a beep. The next training trial then appeared on the screen.

\title{
Subjects
}

Six students, 3 female and 3 female, of University College Cork served as subjects. Their ages ranged from 18 to 25 . All subjects were experimentally naïve, and were nonpsychology majors. Subjects were randomly allocated to one of two experimental conditions.

\section{Procedure}

The procedure of Experiment 3 was identical to Experiment 2 except that the MTS training was modified. On each MTS training trial, the sample stimulus was always presented in the center top half of the computer screen. The comparison stimulus appeared $1.5 \mathrm{~s}$ after the sample stimulus and always appeared directly below the sample, $3 \mathrm{~cm}$ from the lower edge. The sample and comparison stimuli remained on the screen together. Subjects were instructed at the beginning of the experiment to select the comparison by pressing the " $\mathrm{V}$ " key (see instructions below). After a correct response the screen cleared and "Correct' appeared on the screen for $1.5 \mathrm{~s}$, accompanied by a high pitched tone.

\begin{abstract}
At the beginning of this stage of the experiment you will be shown an item on the top of the screen. An item will then appear below this item. Your task is to learn that the item at the top of the screen goes with the item below it. To choose this item, press the marked key " $\mathrm{V}$ " at the center.
\end{abstract}

Press the space-bar twice to continue.

The subject was required to make 12 consecutively correct responses before the subject was allowed to progress to the MTS test.

In Condition 1, each subject received 12 respondent training trials followed by a MTS test. Subjects then received the modified MTS training procedure followed by a MTS test. Condition 2 was identical to Condition 1 except that the subjects received the modified MTS training and MTS test first, followed by the respondent training procedure and a MTS test.

\section{Results and Discussion}

Percent correct on all test trials and mean percent correct calculated across all exposures is presented in Tables 5 and 6 . For both mean percent correct across all exposures and percent correct on the final exposure all 6 subjects produced a greater number of responses in favor of respondent training. In Conditions 1 and 2, on the final exposure all subjects produced $100 \%$ correct responding in favor of respondent training. In the MTS equivalence test, Subjects 13,14 , and 15 of Condition 1 produced $36 \%, 33 \%$, and $38 \%$ correct responses respectively. In Condition 2, Subjects 16, 17, and 18 produced $28 \%, 30 \%$, and $36 \%$ correct responses respectively during the MTS equivalence test. 
Table 5

Respondent Training and Testing Followed by Matching-to-Sample Training and Testing (1 comparison)

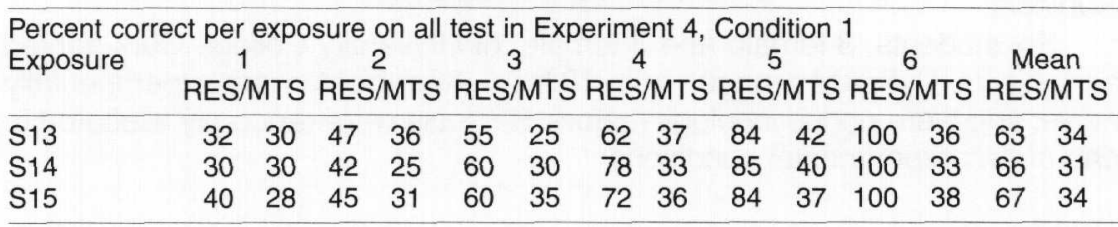

Table 6

Matching-To-Sample Training and Testing Followed by Respondent Training and Testing (1 comparison)

\begin{tabular}{|c|c|c|c|c|c|c|c|c|c|c|c|c|c|c|}
\hline Exposure & MTS & RES & MT & $\begin{array}{l}2 \\
\text { RES }\end{array}$ & MTS & & MTS & $\mathrm{RES}$ & ИT & /RE & MT & $\begin{array}{c}6 \\
\text { S/RE }\end{array}$ & MTS & $\begin{array}{l}\text { ean } \\
\text { /RES }\end{array}$ \\
\hline S16 & 28 & 42 & 30 & 59 & 29 & 65 & 38 & 85 & 38 & 100 & 28 & 10 & 31 & 75 \\
\hline S17 & 25 & 55 & 30 & 65 & 35 & 70 & 40 & 85 & 35 & 95 & 30 & 100 & 32 & 78 \\
\hline S18 & 30 & 35 & 28 & 40 & 25 & 62 & 30 & 85 & 35 & 90 & 36 & 100 & 28 & 68 \\
\hline
\end{tabular}

It was expected that the two training procedures would prove equally effective under the conditions of this experiment, but the respondent training procedure proved again to be more effective than the traditional MTS training procedure. Experiment 3 maybe criticized, however, on the grounds that the correct comparison always appeared in the same position (i.e., centered below the sample), and thus the subject was always required to press the same key ("V") after each samplecomparison presentation. Consequently, subjects could produce consistently correct responses by simply pressing the " $\mathrm{V}$ " key without attending to the contents of the computer screen. Experiment 4 was designed to eliminate this possibility. This experiment was identical to Experiment 3 except that the comparison stimuli appeared, across trials, to the right, center, or left of the screen. When the comparison appeared to the left of the screen the subject was required to press the key " $Z$," when the comparison appeared in the center of the screen the subject was required to press the key "V", and when the comparison appeared to the right of the screen the subject was required to press the key "M."

\section{Experiment 4}

\section{Subject}

Subjects were 6 students, 2 female and 4 male, of University College Cork. Their ages ranged from 18 to 25 . All subjects were experimentally naïve, and were nonpsychology majors. Subjects were randomly allocated to one of two experimental conditions. 


\section{Procedure}

Experiment 4 was identical to Experiment 3 except that the comparison stimuli appeared to right, center, or left of the screen and three response keys were used (i.e., $Z, V$, and $M$ ). When a comparison appeared to the left, center, or right of the screen, pressing the $Z, V$, and $M$ keys, respectively, produced the "Correct" feedback. Pressing any other key produced the "Wrong" feedback (e.g., pressing " $V$ " when the correct comparison appeared on the left or the right). At the beginning of the modified MTS training the following instructions appeared on the screen.

In this experiment you will be shown an item at the top of the screen. Another item will then appear below this item, and will be positioned either on the right, center or the left of the screen. Your task is to learn that the item at the top of the screen goes with the item below it. To choose the item at the left of the screen, press the key marked on the left. To choose the item at the center of the screen, press the key marked on the center. To choose the item at the tight of the screen, press the key marked on the right. Press the space-bar twice to continue.

Subjects were required to make 12 consecutively correct responses before progressing to the MTS test.

In Condition 1 of this experiment subjects were first exposed to the respondent training procedure followed by a MTS test. Subjects were then exposed to the modified MTS procedure followed by a MTS test. Condition 2 was identical to Condition 1 except that subjects were first exposed to the modified MTS training procedure.

\section{Results and Discussion}

Percent correct on all test probes and mean percent correct calculated across all exposures is presented in Tables 7 and 8 . All 6 subjects produced correct responding on their final exposure for both respondent and MTS training. Although all subjects were successful on both training procedures the acquisition of correct responding was faster during respondent training than during MTS training. Subjects 20 and 23 produced accurate responding on their third exposures to the respondent training and testing, Subjects 19 , 21 , and 22 on their fourth exposures, while Subject 24 required six exposures. All 6 subjects required the maximum of six exposures to attain perfect responding during MTS training and testing. For mean percent correct all 6 subjects produced a marginally greater number of correct responses in favor of respondent training.

Under the conditions of this experiment both procedures succeeded in producing accurate responding. Thus the removal of the two incorrect comparisons during MTS training, and varying the position of the correct comparison, appeared to render it as effective as the respondent training procedure, although the latter produced relatively rapid derived responding in comparison to the former. 
Table 7

Respondent Training and Testing Followed by Matching-To-Sample Training Testing (1 comparison, three response keys)

\begin{tabular}{|c|c|c|c|c|c|c|c|c|c|c|c|c|c|c|}
\hline \multicolumn{15}{|c|}{ Percent correct per exposure on all tests in Experiment 5, Condition 1} \\
\hline Exposure & \multicolumn{2}{|c|}{ RES/MTS } & BFC & $2 / \mathrm{MTS}$ & RES & $\begin{array}{l}3 \\
5 / M T S\end{array}$ & RES & 4 & RES & MTS & RES & 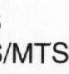 & & $\begin{array}{l}\text { an } \\
\text { /MTS }\end{array}$ \\
\hline S19 & 40 & 30 & 50 & 42 & 64 & 46 & 100 & 64 & 100 & 74 & 100 & 100 & 75 & 59 \\
\hline $\mathrm{S} 20$ & 25 & 32 & 41 & 40 & 100 & 50 & 100 & 54 & 100 & 60 & 100 & 100 & 78 & 56 \\
\hline $\mathrm{S} 21$ & 50 & 41 & 54 & 48 & 60 & 50 & 100 & 60 & 100 & 86 & 100 & 100 & 77 & 64 \\
\hline
\end{tabular}

Table 8

Matching-To-Sample Training and Testing Followed by Respondent Training and Testing (1 comparison, three response keys)

\begin{tabular}{|c|c|c|c|c|c|c|c|c|c|c|c|c|}
\hline Exposur & MTS & L & VI & & & $\begin{array}{l}3 \\
\text { S/RE }\end{array}$ & & & & $\begin{array}{l}5 \\
\text { /RES }\end{array}$ & 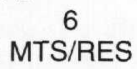 & $\begin{array}{c}\text { Mean } \\
\text { MTS/RES }\end{array}$ \\
\hline 22 & 25 & 35 & 39 & 45 & 47 & 54 & 69 & 100 & 84 & 100 & 100100 & 6172 \\
\hline 23 & 30 & 38 & 42 & 50 & 50 & 100 & 64 & 100 & 75 & 100 & 100100 & $60 \quad 81$ \\
\hline$S 24$ & 33 & 40 & 40 & 42 & 52 & 61 & 71 & 70 & 85 & 86 & 100100 & 6366 \\
\hline
\end{tabular}

\section{General Discussion}

The current study clearly demonstrated (as did Leader et al., 1996; Leader, Smeets, \& Barnes, 2000; Smeets, Leader, \& Barnes, 1997) that it is possible to produce reliable equivalence responding using a respondent training procedure.

In Experiment 1, 3 subjects produced a result clearly in favor of respondent training, and the 3 remaining subjects responded in accordance with both the respondent and MTS training. In effect, the 3 latter subjects changed their response patterns dependent upon the type of training that preceded the equivalence test, whereas the 3 former subjects produced response patterns that were consistently in accordance with the respondent training. At the end of Experiment 2, the test results indicated that the respondent training procedure facilitated equivalence responding more readily than did the MTS training procedure. However, Experiment 1 could be criticized on the grounds that it departed somewhat from the traditional MTS training. Specifically, in both experiments subjects were exposed to a "criterion-independent" form of MTS training (i.e., subjects were given 60 training trials irrespective of their performance accuracy before moving on to the next stage of the experiment). To circumvent this problem Experiment 2 was conducted.

In this second experiment, subjects were required to produce 12 consecutively correct responses during the MTS training before progressing to the MTS equivalence test. Subjects were previously given 60 respondent training trials, but in this experiment they were given 12 training trials. In effect, the number of consecutively correct MTS training 
trials was matched to the number of respondent training trials. Under the conditions of this experiment the respondent training again proved to be more effective than MTS. In fact, all 6 subjects responded in accordance with respondent training during the MTS test. This is a particularly surprising result in that the subjects were exposed to only one fifth of the respondent training trials used in Experiments 1 and 2 . This result indicated that the important variable in establishing equivalence using a respondent training procedure is not the amount of training trials, per exposure, but the repeated exposure to training and testing (i.e., no subject produced equivalence after one exposure to the training and testing). Indeed, these data are consistent with the results reported in the Leader et al. (1996) study.

The data of Experiment 2 raises the question, what variables make the respondent procedure more effective than the MTS procedure? In the MTS training procedure both positive and negative comparisons are presented on each trial, and thus at least on some occasions a subject will observe the sample stimulus and then immediately observe an incorrect comparison. Even if that comparison is not chosen on that trial, the sample may acquire some of the perceptual functions of the comparison through respondent-type processes. In other words, seeing a sample and then an incorrect comparison may lead a subject to see privately that comparison when subsequently presented with the sample (see Barnes, 1994, for a detailed discussion). This effect, if it occurs, would constitute a form of competing stimulus control during test trials. In Experiment 3 , therefore, we decided to eliminate the potential for competing stimulus control by removing the negative comparisons from the MTS training procedure.

In Experiment 3 the respondent procedure again proved more effective than the modified MTS procedure. The removal of the negative comparisons from the MTS training procedure did not appear to augment the MTS test performance. Experiment 3 could be criticized because the correct comparison always appeared at the center of the screen, and thus the subjects were simply required to press the same key after each sample-comparison presentation. Consequently, subjects may have simply pressed the response key without attending to the stimulus presentations on the screen. Experiment 4 addressed this criticism.

In Experiment 4, the subjects were required to press one of the three marked keys contingent upon the location of the comparison on the screen, thereby facilitating attention to the comparison stimulus on each trial. In this experiment, both training procedures succeeded in producing accurate responding. In effect, the removal of the negative comparisons from the MTS training rendered the MTS training almost as effective as respondent training. These data support the suggestion that the presentation of negative comparisons during the traditional MTS procedure may sometimes provide a source of competing stimulus control over the formation of equivalence classes among positive samplecomparison stimuli. 
In Experiments 1, 2, and 3 performance on test trials following MTS training was surprisingly low. Even when a stability criterion was employed in Experiment 2 and when the negative comparisons were removed in Experiment 3, test performances were still relatively low. However upon inspection of the raw data it appears that students "adopted" respondent training during the MTS test. In effect students responded in accordance with the respondent training during the MTS testing.

The results of the current study highlight a number of avenues for future research. The need for repeated training and testing was significantly reduced by using a simple-to-complex protocol, in which subjects were trained and tested for symmetry relations, before being trained and tested for more complex relations (i.e., transitivity, and combined symmetry and transitivity) (Fields, Adams, Newman, \& Verhave, 1992). Perhaps, therefore, equivalence would have emerged far more rapidly during respondent and MTS training, if subjects had been trained on the three A-B tasks and tested for B-A symmetry; then trained on the B-C tasks and tested for C-B symmetry; then tested for A-C transitivity; and finally tested for C-A combined symmetry and transitivity. It would be interesting, therefore, to examine interactions between training design with respondent and MTS training protocols. Other issues relate to class size. In the current study three 3-member classes were used, but many equivalence studies have used four or more members per class, and/or four or more classes. How would these variables interact with the respondent procedure? Would the superiority of the respondent procedure increase, decrease, or remain constant across different class sizes or numbers of classes. Other researchers have also examined the role of instructions on equivalence formation (Dymond \& Barnes, 1994; Saunders, Saunders, Williams, \& Spradlin, 1993), and thus here again we might examine interaction effects between levels of instruction and training procedure.

Another issue that requires further study is whether continued exposure to the training and testing phases of Experiment 2 would produce responding in accordance with both the respondent and MTS procedures. In the current study, the experiment was terminated whenever subjects responded consistently following both types of training or after six exposures. The results showed a clear superiority for the respondent procedure, but perhaps this was temporary rather than permanent. That is, perhaps the respondent procedure produced derived relational responding more rapidly than MTS, and thus continued exposure to the experimental procedures would have eventually produced equivalence responding in accordance with both types of training. From the point of view of understanding the behavioral processes at work here, it may be that equivalence classes were formed relatively quickly with the respondent procedure, and were thus difficult to modify with the less rapid MTS procedure. This interpretation would certainly be consistent with the previous research that has shown that it is difficult to modify previously established derived relations (Pilgrim \& Galizio, 1990, 1995; Roche et al., 1997). 
The superiority of the respondent training procedure may have ramification in the applied setting. Indeed in a recent study, Leader and Barnes-Holmes (2001) used the respondent training procedure to establish equivalence relations among fractions, decimals, and pictorial representations of these numerical properties, with 5-year-old children in an educational setting. There was also evidence of stimulus generalization involving novel pictorial representations that were physically similar. Given these findings it may now be possible to use the respondent procedure in other areas of research in the applied domain.

Addressing these and possibly other issues raised by the current study would shed even further light on the equivalence effect itself, and would help to identify those key variables that either facilitate or interfere with the formation of equivalence classes in laboratory settings. Clearly, such a program of research would also have important applied implications. Only by systematically tracking down the controlling variables over equivalence class formation by comparing and contrasting different training and testing procedures in combination with other variables (such as training protocols and level of instruction) will we be in a position to design the most effective educational interventions for applied settings.

\section{References}

BARNES, D. (1994). Stimulus equivalence and relational frame theory. The Psychological Record, 44, 91-124.

DYMOND, S., \& BARNES, D. (1994). A transfer of self-discrimination response functions through equivalence relations. Journal of the Experimental Analysis of Behavior, 62, 251-267.

FIELDS, L., ADAMS, B. J., NEWMAN, S., \& VERHAVE, T. (1992). Interactions among emergent relations during equivalence class formation. The Quarterly Journal of Experimental Psychology, 45B, 125-138.

FIELDS, L., ADAMS, B. J., \& VERHAVE, T. (1993). The effects of equivalence class structure on test performances. The Psychological Record, 43, 697-712.

FIELDS, L., \& VERHAVE, T. (1987). The structure of equivalence classes. Journal of the Experimental Analysis of Behavior, 48, 317-332.

HAYES, S. C. (1991). A relational control theory of stimulus equivalence. In L. J. Hayes \& P. N. Chase (Eds.), Dialogues of verbal behavior (pp. 19-40). Reno, NV: Context Press.

HEALY, O., BARNES, D., \& SMEETS, P. M. (1998). Derived relational responding as an operant: The effects of between-session feedback. The Psychological Record, 48, 511-536.

LEADER, G., \& BARNES-HOLMES, D. (2001). Establishing fraction-decimal equivalence using a respondent-type training procedure. The Psychological Record, 51, 151-165.

LEADER, G., BARNES, D., \& SMEETS, P. M. (1996). Establishing equivalence relations using a respondent-type training procedure. The Psychological Record, 46, 685-706. 
LEADER, G., SMEETS, P. M., \& BARNES, D. (2000). Establishing equivalence relations using a respondent-type training procedure III. The Psychological Record, 50, 63-78.

PILGRIM, C., \& GALIZIO, M. (1990). Relations between baseline contingencies and equivalence probe performances. Journal of the Experimental Analysis of Behavior, 54 213-224.

PILGRIM, C., \& GALIZIO, M. (1995). Reversal of baseline relations and stimulus equivalence: I. Adults. Journal of the Experimental Analysis of Behavior, 63, 225-238.

ROCHE, B., BARNES, D., \& SMEETS, P. (1997). Incongruous stimulus pairings and conditional discrimination training: Effects on relational responding. Journal of the Experimental Analysis of Behavior, 68, 143-160.

SAUNDERS, R. R., \& GREEN, G. (1999). A discrimination analysis of trainingstructure effects on stimulus equivalence outcomes. Journal of the Experimental Analysis of Behavior, 72, 117-137.

SAUNDERS, K. J., SAUNDERS, R. R., WILLIAMS, D. W., \& SPRADLIN, J. E. (1993). An interaction of instructions and training design on stimulus class formation: Extending the analysis of equivalence. The Psychological Record, 43, 725-744.

SIDMAN, M. (1971). Reading and auditory-visual equivalence. Journal of Speech and Hearing Research, 14, 5-13.

SIDMAN, M. (1980). A note on the measurement of conditional discrimination. Journal of the Experimental Analysis of Behavior, 33, 285-289.

SIDMAN, M. (1986). Functional analysis of emergent and verbal classes. In T. Thompson \& M. A. Zeiler (Eds.), Analysis and interaction of behavioral units (pp. 213-245). Hillsdale, NJ: Erlbaum.

SIDMAN, M. (1987). Two choices are not enough. Behavior Analysis, 22, 11-18.

SIDMAN, M., \& CRESSON, O. (1973). Reading and crossmodal transfer of stmulus equivalence in severe retardation. American Journal of Mental Deficiency, 77, 513-523.

SIDMAN, M., \& TAILBY, W. (1982). Conditional discrimination vs. matching-tosample: An expansion of the testing paradigm. Journal of the Experimental Analysis of Behavior, 37, 5-22.

SMEETS, P. M., LEADER, G., \& BARNES, D. (1997). Establishing stimulus classes in adults and children using a respondent-type training procedure: A follow-up study. The Psychological Record, 47, 285-308.

WULFERT, E., DOUGHER, M. J., \& GREENWAY, D. E. (1991). Protocol analysis of the correspondence of verbal behavior and equivalence class formation. Journal of the Experimental Analysis of Behavior, 56, 489-504. 